\title{
Progesterone Resistance, Aromatase, and Inflammation: The Important Relationships Between Hormones and Inflammation
}

\author{
Paola Vigano • Elisa Rabellotti • Luca Pagliardini • \\ Edgardo Somigliana • Massimo Candiani • \\ Paolo Vercellini
}

Published online: 27 April 2012

(C) Springer Science+Business Media, LLC 2012

\begin{abstract}
Endometriosis is a complex gynecologic disorder in which alterations of specific biological processes involving both the endocrine and the immune systems have been identified. Recently, a reduced endometrial responsiveness to progesterone has been suggested as a crucial element in the pathophysiology of the disease but it is unclear whether this reduced sensitivity to the steroid is involved in the disease induction or is simply a consequence of the disease condition. Indeed, endometriosis is associated with a local and systemic inflammatory process that is strongly interrelated to the steroid activity, and both the systems mutually interact in a complex loop that strongly influences the disease development and maintenance. The link between prostaglandin $\mathrm{E}_{2}\left(\mathrm{PGE}_{2}\right)$, aromatase activity, and local estrogen synthesis represents a paradigm of this loop. A better understanding of the pathophysiology of this interaction should enable researchers to develop targeted therapeutics that may limit this self-supporting pathologic loop.
\end{abstract}

Keywords Progesterone · Progesterone resistance ·

Aromatase $\cdot$ Endometriosis $\cdot$ Inflammation

P. Vigano $(\varangle) \cdot$ E. Rabellotti $\cdot$ L. Pagliardini $\cdot$ M. Candiani

Obstetrics and Gynecology Unit,

San Raffaele Scientific Institute,

Milano, Italy

e-mail: vigano.paola@hsr.it

E. Somigliana

Department of Obstetrics, Gynecology and Neonatology,

Fondazione Cà Granda, Ospedale Maggiore Policlinico,

Milano, Italy

P. Vercellini

Istituto Ostetrico e Ginecologico "L. Mangiagalli,"

University of Milano,

Milano, Italy

\section{Introduction}

Endometriosis is an estrogen-dependent disorder, defined as the presence of endometrial tissue outside of the uterus in lesions of varying sizes and appearance containing endometrial glands and stroma. Symptoms arise from cyclical bleeding into the surrounding tissues, which results in inflammation and formation of scarring and adhesions potentially causing infertility. Signs and symptoms may include painful periods (dysmenorrhea), pelvic pain not associated with menses, painful intercourse (dyspareunia), painful urination (dysuria), and painful bowel movements. It may be asymptomatic or associated with symptoms of pain and/or infertility $[1 \bullet \bullet]$. However, endometriosis is much more complicated than either lesions or symptoms, and because of this complexity, its classification is evolving from a local disorder to a chronic systemic condition.

Although described in the literature for more than 300 years [2], endometriosis remains a mysterious and elusive disease that can impact on the general physical, mental, and social well being of a woman and can have a profound effect on the woman's life. From a pathogenetic point of view, the disease inheritance is thought to be polygenic with a complex, multifactorial etiology. The gold standard for the diagnosis of pelvic disease is surgical assessment during laparoscopy [3]. Surgical removal of endometriotic implants improves fertility and commonly provides temporary relief for pain symptoms. However, the cumulative recurrence rates after surgery vary greatly depending on the degree of endometriosis and possibly also on the extent of surgery [4]. Medical treatments aimed at hormone suppression are effective for pain but their cost and side effect profile varies [3]. Moreover, they induce a hypoestrogenic state and can be used only for a limited time owing to important and sometimes unacceptable side effects [5]. 
Endometriosis long has been considered a surgical gynecological disease. Currently, there is a need for clinical management of the disease by multidisciplinary teams that address medical, surgical, and psychological issues. Moreover, endometriosis is still an unmet clinical need because an optimal drug that allows for both pain management and continued attempts to conceive does not exist. Therefore, a great effort is directed toward the understanding of the basic mechanisms underlying the disease to identify an ideal treatment that would eliminate endometriotic lesions, prevent recurrence, and not impede ovulation. The interrelationship between the inflammatory process associated with the disease and the altered response to steroid hormones may represent an area of interest in this context.

\section{Local Inflammatory Processes}

There is a general agreement that a sterile, local inflammation occurs in the peritoneal cavity of women with endometriosis and that an altered function of immune-related cells characterizes the peritoneal environment [6]. Interestingly, based on mouse model of endometriosis, both macrophages in the peritoneal fluid and macrophages infiltrating ectopic lesions display features of alternative activation, suggesting that signals in the peritoneal cavity of endometriotic patients and restricted to the ectopic tissue switch the differentiation program of endogenous blood-derived precursors toward an alternative reparative phenotype [7]. Adoptively transferred, alternatively activated M2 macrophages dramatically enhance endometriotic lesion growth through the secretion of different arrays of soluble factors. Various cytokines and growth factors are indeed increased in peritoneal fluid of women with endometriosis, and in the setting of chronic local tissue destruction, it is also likely that autoreactivity develops. For instance, interleukin-8 and monocyte chemotactic peptide-1 (MCP-1) are found to be elevated in the peritoneal fluid of women with endometriosis and levels have been correlated with the severity of the disease [6].

Therefore, endometriosis itself does favor a peritoneal inflammatory situation that contributes to maintaining the disease. Moreover, macrophages are important for maintaining the viability of newly formed vessels and represent a potential therapeutic target in endometriosis. Tie2expressing macrophages (TEMs) have nonredundant functions in promoting angiogenesis and growth of experimental tumors and they have been shown to infiltrate areas surrounding newly formed endometriotic blood vessels and endometriotic lesions. TEM depletion arrested the growth of established lesions without toxicity. Lesion architecture was disrupted with (1) loss of glandular organization, (2) reduced neovascularization, and (3) activation of caspase 3 in $\mathrm{CD} 31(+)$ endothelial cells [8•].

\section{Abnormal Immune Response at Systemic Level}

In recent years, there have been discussions suggesting that endometriosis might even be regarded as an autoimmune disease [6]. Some investigators have reported common clinical elements between patients with endometriosis and patients with various autoimmune processes $[9,10]$, whereas others have reported that endometriosis exhibits a gene expression signature reminiscent of autoimmune disorders [11]. Endometriosis lesions are characterized by the presence of abundant plasma cells, and one of the most upregulated cytokines in these lesions is BlyS, a cytokine known to be critical for normal B-cell development; high levels of BlyS overstimulate various B cell responses, leading to the initiation and exacerbation of autoimmune responses [12].

In support for an abnormal development of immune response at systemic level as well, specific genes involved in leukocyte activation such as interleukin-2 receptor gamma (IL-2RG), were shown to be differentially expressed in blood leukocytes of women with endometriosis compared to those present in control women [13]. Finally, based on population-based epidemiological studies, women with endometriosis have an increased risk for non-Hodgkin's lymphoma, thus suggesting an underlying immune system-associated pathology and a more generalized dysfunction $[14,15]$.

Notwithstanding these findings supporting the critical role of immunologic/inflammatory phenomena in the natural history of endometriosis, two important issues are still to be clarified: (1) whether these events are only an epiphenomenon or are causally related to the disease and more importantly, (2) to what extent these events may affect the systemic immunologic status.

Recently, we performed a large-scale gene expression study on peripheral blood mononuclear cells (PBMCs) from endometriosis patients, comparing gene expression profile in the severe diseased stage with the profile established after a conventional surgical treatment for removal of endometriotic lesions [16]. Genes identified in PBMCs as downregulated after the surgical intervention, and thus potentially induced by the disease presence, were shown to be common to those identified with a similar approach in psoriasis, a nongynecologic chronic inflammatory diseases [17], strongly indicating a relevant influence of endometriosis on systemic immune regulatory molecules. Among the genes identified, Pre-B-cell colony enhancing factor 1 (PBEF), dual specificity phosphatase 1 (DUSP1), FOS, RHOB, and S100P. PBEF has been implicated in the pathogenesis of a number of different human diseases that share an inflammatory basis such as rheumatoid arthritis and type 2 diabetes [18]. DUSP1, FOS, RHOB, and S100P were identified by transcriptional profiling in atrial myocardial samples after a cardiopulmonary bypass (CPB) that is known to induce 
mediators of the inflammatory response [19]. Based on all these findings, the impact of endometriosis at systemic level should be recognized as more significant than previously envisioned and the possible consequences of this influence should be considered in relation to the long-term effects of a chronic systemic inflammation.

\section{Evidence for an Inflammation-Associated Progesterone Resistance}

Progesterone is a master regulator of endometrial tissue and it is estimated to regulate expression of hundreds of genes during the various phases of the menstrual cycle. Two natural isoforms of progesterone receptors (PRs), PR-A and PR-B, are coexpressed in all normal progesterone target tissues and the human PR-A isoform differs from the PR-B isoform because it lacks the first 164 amino acids contained in the PR-B. The physiological importance of maintaining the correct relative expression levels of PR isoforms in tissues is indicated by detection of aberrant ratios of PR isoforms in human endometrial and breast cancers [20]. In this type of cancer cells, alteration of the PR-A/PR-B ratio is known to favor cellular invasion and metastasis [21,22]. Overexpressed PR-B levels are often found in highly malignant endometrial cancers and selective ablation of the A isoform in knockout mice results in endometrial hyperproliferative and premalignant changes [23]. Recently, the equal PR-A to PR-B ratio of the normal endometrium has been suggested to be extensively deregulated also in the endometriotic tissue, leading to an impaired stromal differentiation and a consequent relative resistance to progesterone action in endometriosis [24-27]. Studies addressing progesterone resistance in endometriosis are summarized in Table 1.

According to Attia et al. [24], the progesterone resistance in the endometriotic tissue can be explained by the complete absence of PR-B transcripts and protein and the presence of PR-A in ectopic lesions. Similar findings have been reported in epithelial cells selected from a small number of ectopic samples [26]. Moreover, because a number of progesterone target genes are deregulated also in the eutopic endometrium of women with endometriosis [28], there has been suggestive indications that endometrium of affected women could reflect the alteration found in the endometriotic lesions. In keeping with this idea, PR-B expression was shown to be statistically lower in endometrium from women with endometriosis as compared with that of normal women at both protein [25] and messenger RNA (mRNA) level [26]. Conversely, more recently, Bukulmez et al. [27] failed to find any significant difference in PR-A and PR-B mRNA expression between control endometrium and eutopic endometrium of women affected, although confirming a significant reduction of both PR isoforms in endometriomas. Our results are completely in line with those obtained by Bukulmez et al. [27] for the eutopic endometrium showing no differences in PR-A and PR-B expression between endometrial stromal cells derived from women with and without endometriosis at both mRNA and protein level [29]. On the other hand, in a previous study [30], we have demonstrated that in cells from women with endometriosis but not from those without the disease, progesterone can induce endometrial stromal cell migration and cytoskeleton rearrangement, two events that occur during cell invasion. Therefore, based on the data reported so far, it has been hypothesized that a reduced PR-B expression might be responsible for elevated local estradiol levels, impaired differentiation, deficient apoptosis, and increased cell invasion observed in the pathologic tissue. On this basis, the efficacy of endometriotic lesion suppression with progestins has been recently questioned based on this purported progesterone resistance in both eutopic and ectopic endometrium of women with the disease. This would explain why about $9 \%$ of patients do not respond to progestin treatment [4].

However, within the context of basic research on endometriosis, it is often difficult to distinguish between a primary etiologic factor and alterations occurring with time or under peculiar hormonal and inflammatory conditions at both transcriptional and nontranscriptional levels. The inflammatory environment constitutes, in itself, a trigger for epigenetic reprogramming (eg, through local extracellular acidosis and deposit of reactive substances). Reactive halogen compounds, which are a byproduct of many chemical
Table 1 Studies addressing and relating to progesterone response in endometriosis

\begin{tabular}{lll}
\hline $\begin{array}{l}\text { Altered progesterone } \\
\text { sensitivity }\end{array}$ & Genetic evidence & Near et al. [58] \\
& Direct phenotype evidence & Attia et al. [24], Igarashi et al. [25], \\
& Wu et al. [26], Bukulmez et al. [27], \\
& Aghajanova et al. [59], Gentilini et al. [29] \\
& Indirect phenotype evidence & Burney et al. [28], Kao et al. [60], \\
& Bulun et al. [61], Aghajanova et al. [62] \\
Functional evidence & Bruner-Tran et al. [63], Nayyar et al. [64], \\
& Gentilini et al. [30] \\
Clinical evidence & Vercellini et al. [65] \\
\hline
\end{tabular}


reactions produced by inflammatory processes, cause DNA methylation alteration. Chemical transformations of DNA methylation contributes to disruption of the epigenetic code, which could result in a disturbed readout by the methylation-binding proteins involved in both activating and silencing genes [31]. Therefore, it cannot be excluded that secondary events, such as the increased inflammatory response observed in ectopic endometrium, may contribute to the differential receptor expression pattern demonstrated in some endometriotic lesions.

Finally, it should be considered that exposure to environmental endocrine disruptors in utero and during neonatal life can lead to epigenetic modification of numerous genes critical to reproductive tract development and function [32, 33]. Recent evidence suggests that genes that may impact a woman's risk for endometriosis may be among them, for instance the PR [4, 34-37]. Indeed, an endometriosis-like phenotype in mice both at histologic and molecular level can be elicited by early, developmental exposure to endocrine disruptors [36, 38].

Therefore, the origin and the mechanisms underlying the supposed progesterone resistance in endometriosis require further investigation.

\section{Targeting the "Aromatase" Enzyme for Treatment}

Endometriosis requires estrogen for its continued growth, and if deprived of these hormones, it tends to regress. Aromatase is a cytochrome P450 enzyme that catalyzes the rate-limiting step in estrogen biosynthesis, the conversion of androgens to estrogens [39]. The enzyme is expressed by many human cell types including ovarian granulosa cells, placental syncytiotrophoblasts, adipose cells, and skin fibroblasts. Estrogen action is classically believed to occur via an endocrine mechanism. Studies on aromatase expression in breast cancer demonstrated that paracrine mechanisms play an important role in estrogen action in this tissue. Estrogens also display an "intracrine" effect; estrogens produced by aromatase activity in the cytoplasm of leiomyoma smooth muscle cells or in endometriotic stromal cells can exert their effects by readily binding to their nuclear receptor within the same cell [39]. It has been suggested, albeit not consistently [40,41], that aromatase is expressed at higher levels in endometriosis implants than in normal endometrium, thus providing the ectopic mucosa with excessive proliferative stimulus [42-44]. Prostaglandin $\mathrm{E}_{2}\left(\mathrm{PGE}_{2}\right)$ was identified as the most potent inducer of aromatase activity in endometriotic cells, and estrogen, in turn, was found to upregulate $\mathrm{PGE}_{2}$ formation by stimulating cyclooxygenase type 2 enzyme. Thus, a positive feedback loop for continuous local estrogens and $\mathrm{PGE}_{2}$ production is established in the pathologic tissue itself, possibly favoring the proliferative and inflammatory characteristics of endometriosis. These findings suggest that the aberrant expression of aromatase in endometriotic tissue might be involved in the pathogenetic mechanisms of this disease promoting survival and growth of the disease lesions. The mechanisms that mediate the regulation of aromatase activity in endometriotic tissue also have been investigated. The most critical mechanism is mediated by the aberrantly expressed key transcriptional enhancer SF-1 in endometriotic tissue. SF-1 binds to a specific response element in the promoters of a number of steroidogenic genes in the ovary and mediates the responsiveness of a portion of these genes to cyclic adenosine monophosphate (cAMP). In endometriotic stromal cells, $\mathrm{PGE}_{2}$ induces coordinate binding of SF-1 to the promoters of steroidogenic acute regulatory protein (StAR) and aromatase gene to co-activate their expression [39]. SF-1 is expressed specifically in endometriosis but not in eutopic endometrium due to an epigenetic mechanism that permits binding of activator versus inhibitor complexes to its promoter. These observations represent the molecular bases for use of aromatase inhibitors to treat endometriosis [45] and have prompted performance of several pilot studies using one of the two available reversible aromatase inhibitors (ie, anastrozole and letrozole), which compete with androgens for aromatase-binding sites [46, 47]. Aromatase inhibitors have been tested in surgically induced models of endometriosis in mice. The administration of letrozole ( $10 \mu \mathrm{g} /$ day subcutaneously) for 4 weeks to wild-type mice demonstrated a dose-dependent suppressive effect on the growth of ectopic tissue [48].

However, again it is unclear if the increased expression of this cytochrome P-450 complex constitutes the very reason for survival and proliferation of regurgitated endometrial cells, or if it is the consequence of local inflammation, because of prostaglandins [49], in which case, aromatase inhibitors would simply treat an epiphenomenon. Moreover, in humans, the premenopausal ovary is generally considered to be resistant to blockade of estrogen production by aromatase inhibitors because any lowering of plasma estrogen levels would cause reflexive increases in gonadotrophins [50]. This would then induce increased ovarian production of estrogens. Indeed, aromatase inhibitors have been suggested to correct ovulatory dysfunction as well as to increase the number of follicles in controlled ovarian stimulation protocols [51]. Therefore, in premenopausal women, these compounds must be used together with other drugs (eg, gonadotropin-releasing agonists, danazol, oral contraceptives [OCs], and progestins) that could effectively suppress gonadotropins and reduce ovarian activity [52-54]. Clearly, when these combined regimens are used for endometriosis, it is impossible to assess how much of the effect on pain is related to which of the two medications [55]. When the efficacy of letrozole plus norethisterone acetate (NETA) was compared with that of NETA 
alone in women with rectovaginal endometriosis [56], the reported intensity of pelvic pain was slightly lower in the combined regimen group, but owing to the side effects of letrozole (mainly joint pain and myalgia), satisfaction with treatment was higher in patients who used NETA alone. After treatment discontinuation, symptoms recurred without significant between-group differences, demonstrating that also aromatase inhibitors are neither cytoreductive nor curative. In fact, histological examination of endometriotic lesions excised after treatment with letrozole shows preservation of endometrial glands and high stromal proliferative activity [55].

Aromatase inhibitors seem effective in treating severe postmenopausal endometriosis through blockade of extraovarian estrogen production [57], but their use in premenopausal endometriosis should be considered with caution [47].

\section{Conclusions}

Endometriosis is a common condition with a variety of different phenotypes. The disease has been associated with progesterone resistance, and recently, this reduced sensitivity to this steroid has been linked to the inflammatory process associated with the disease. It remains unclear whether this progesterone resistance contributes to the original development of the condition or reflects the changes in endocrine-immune relationships that evolve as a consequence of the disease progression. In any case, these endocrine-immune changes may represent novel targets for disease treatment. Although it has been demonstrated to be clinically less efficacious than originally envisioned, the targeting of the aromatase enzyme represents a paradigm of this approach. Reducing the impact of the inflammatory environment that may affect the progression of endometriosis or improving endometrial response at both eutopic and ectopic sites will be key to the development of better therapeutic strategies for this disease.

Disclosures No potential conflicts of interest related to this article were reported.

\section{References}

Papers of particular interest, published recently, have been highlighted as:

- Of Importance

-. Of major importance

1. •• Vercellini P, Crosignani P, Somigliana E, et al. 'Waiting for Godot': a commonsense approach to the medical treatment of endometriosis. Hum Reprod. 2011;26:3-13. This is an up-to-date critical overview on the current treatments for endometriosis.

2. Knapp VJ. How old is endometriosis? Late 17th- and 18th-century European descriptions of the disease. Fertil Steril. 1999;72:10-4.

3. Kennedy S, Bergqvist A, Chapron C, et al. ESHRE guideline for the diagnosis and treatment of endometriosis. Hum Reprod. 2005;20:2698-704.

4. Guo SW. Recurrence of endometriosis and its control. Hum Reprod Update. 2009;15:441-61.

5. Crosignani $\mathrm{P}$, Olive D, Bergqvist A, Luciano A. Advances in the management of endometriosis: an update for clinicians. Hum Reprod Update. 2006;12:179-89.

6. Barrier BF. Immunology of endometriosis. Clin Obstet Gynecol. 2010;53:397-402.

7. Bacci M, Capobianco A, Monno A, et al. Macrophages are alternatively activated in patients with endometriosis and required for growth and vascularization of lesions in a mouse model of disease. Am J Pathol. 2009;175:547-56.

8. Capobianco A, Monno A, Cottone L, et al. Proangiogenic Tie(2) macrophages infiltrate human and murine endometriotic lesions and dictate their growth in a mouse model of the disease. Am J Pathol. 2011;179:2651-9. This article is a characterization of macrophages infiltrating endometriotic lesions in terms of angiogenic potential.

9. Nothnick WB. Treating endometriosis as an autoimmune disease. Fertil Steril. 2001;76:223-31.

10. Sinaii N, Cleary SD, Ballweg ML, et al. High rates of autoimmune and endocrine disorders, fibromyalgia, chronic fatigue syndrome and atopic diseases among women with endometriosis: a survey analysis. Hum Reprod. 2002;17:2715-24.

11. Matarese G, De Placido G, Nikas Y, Alviggi C. Pathogenesis of endometriosis: natural immunity dysfunction or autoimmune disease? Trends Mol Med. 2003;9:223-8.

12. Hever A, Roth RB, Hevezi P, et al. Human endometriosis is associated with plasma cells and overexpression of B lymphocyte stimulator. Proc Natl Acad Sci U S A. 2007;104:12451-6.

13. Flores I, Rivera E, Mousses S, et al. Identification of molecular markers for endometriosis in blood lymphocytes by using deoxyribonucleic acid microarrays. Fertil Steril. 2006;85:1676-83.

14. Somigliana E, Vigano P, Parazzini F, et al. Association between endometriosis and cancer: a comprehensive review and a critical analysis of clinical and epidemiological evidence. Gynecol Oncol. 2006;101:331-41.

15. Vigano P, Somigliana E, Parazzini F, Vercellini P. Bias versus causality: interpreting recent evidence of association between endometriosis and ovarian cancer. Fertil Steril. 2007;88:588-93.

16. Gentilini D, Perino A, Vigano P, et al. Gene expression profiling of peripheral blood mononuclear cells in endometriosis identifies genes altered in non-gynaecologic chronic inflammatory diseases. Hum Reprod. 2011;26:3109-17.

17. Koczan D, Guthke R, Thiesen HJ, et al. Gene expression profiling of peripheral blood mononuclear leukocytes from psoriasis patients identifies new immune regulatory molecules. Eur J Dermatol. 2005;15:251-7.

18. Luk T, Malam Z, Marshall JC. Pre-B cell colony-enhancing factor (PBEF)/visfatin: a novel mediator of innate immunity. J Leukoc Biol. 2008;83:804-16.

19. Voisine P, Ruel M, Khan TA, et al. Differences in gene expression profiles of diabetic and nondiabetic patients undergoing cardiopulmonary bypass and cardioplegic arrest. Circulation. 2004;110: II280-286.

20. Mote PA, Bartow S, Tran N, Clarke CL. Loss of co-ordinate expression of progesterone receptors $\mathrm{A}$ and $\mathrm{B}$ is an early event in breast carcinogenesis. Breast Cancer Res Treat. 2002;72:163-72.

21. Ito K, Utsunomiya H, Yaegashi N, Sasano H. Biological roles of estrogen and progesterone in human endometrial carcinoma-new 
developments in potential endocrine therapy for endometrial cancer. Endocr J. 2007;54:667-79.

22. De Vivo I, Huggins GS, Hankinson SE, et al. A functional polymorphism in the promoter of the progesterone receptor gene associated with endometrial cancer risk. Proc Natl Acad Sci U S A. 2002;99:12263-8.

23. Jacobsen BM, Richer JK, Sartorius CA, Horwitz KB. Expression profiling of human breast cancers and gene regulation by progesterone receptors. J Mammary Gland Biol Neoplasia. 2003;8:25768 .

24. Attia GR, Zeitoun K, Edwards D, et al. Progesterone receptor isoform A but not B is expressed in endometriosis. J Clin Endocrinol Metab. 2000;85:2897-902.

25. Igarashi TM, Bruner-Tran KL, Yeaman GR, et al. Reduced expression of progesterone receptor-B in the endometrium of women with endometriosis and in cocultures of endometrial cells exposed to 2,3,7,8-tetrachlorodibenzo-p-dioxin. Fertil Steril. 2005;84:6774.

26. Wu Y, Strawn E, Basir Z, et al. Promoter hypermethylation of progesterone receptor isoform $\mathrm{B}$ (PR-B) in endometriosis. Epigenetics. 2006;1:106-11.

27. Bukulmez O, Hardy DB, Carr BR, et al. Inflammatory status influences aromatase and steroid receptor expression in endometriosis. Endocrinology. 2008;149:1190-204.

28. Burney RO, Talbi S, Hamilton AE, et al. Gene expression analysis of endometrium reveals progesterone resistance and candidate susceptibility genes in women with endometriosis. Endocrinology. 2007;148:3814-26.

29. Gentilini D, Vigano P, Vignali M, et al. Endometrial stromal progesterone receptor-A/progesterone receptor-B ratio: no difference between women with and without endometriosis. Fertil Steril. 2010;94:1538-40.

30. Gentilini D, Vigano P, Somigliana E, et al. Endometrial stromal cells from women with endometriosis reveal peculiar migratory behavior in response to ovarian steroids. Fertil Steril. 2010;93:70615 .

31. Backdahl L, Bushell A, Beck S. Inflammatory signalling as mediator of epigenetic modulation in tissue-specific chronic inflammation. Int J Biochem Cell Biol. 2009;41:176-84.

32. Bromer JG, Zhou Y, Taylor MB, et al. Bisphenol-A exposure in utero leads to epigenetic alterations in the developmental programming of uterine estrogen response. FASEB J. 2010;24:2273-80.

33. Heindel JJ, McAllister KA, Worth Jr L, Tyson FL. Environmental epigenomics, imprinting and disease susceptibility. Epigenetics. 2006;1:1-6.

34. Bruner-Tran KL, Osteen KG. Developmental exposure to TCDD reduces fertility and negatively affects pregnancy outcomes across multiple generations. Reprod Toxicol. 2011;31:344-50.

35. Bruner-Tran KL, Ding T, Osteen KG. Dioxin and endometrial progesterone resistance. Semin Reprod Med. 2010;28:59-68.

36. Nayyar T, Bruner-Tran KL, Piestrzeniewicz-Ulanska D, Osteen KG. Developmental exposure of mice to TCDD elicits a similar uterine phenotype in adult animals as observed in women with endometriosis. Reprod Toxicol. 2007;23:326-36.

37. Stilley JA, Woods-Marshall R, Sutovsky M, et al. Reduced fecundity in female rats with surgically induced endometriosis and in their daughters: a potential role for tissue inhibitors of metalloproteinase 1. Biol Reprod. 2009;80:649-56.

38. Signorile PG, Spugnini EP, Mita L, et al. Pre-natal exposure of mice to bisphenol A elicits an endometriosis-like phenotype in female offspring. Gen Comp Endocrinol. 2010;168:318-25.

39. Seli E, Berkkanoglu M, Arici A. Pathogenesis of endometriosis. Obstet Gynecol Clin North Am. 2003;30:41-61.
40. Colette S, Lousse JC, Defrere S, et al. Absence of aromatase protein and mRNA expression in endometriosis. Hum Reprod. 2009;24:2133-41.

41. Colette S, Donnez J. Endometriosis. N Engl J Med. 2009;360:19112. author reply 1912.

42. Attar E, Bulun SE. Aromatase inhibitors: the next generation of therapeutics for endometriosis? Fertil Steril. 2006;85:1307-18.

43. Bulun SE. Endometriosis. N Engl J Med. 2009;360:268-79.

44. Bulun SE ZK, Takayama K, Noble L, Michael D, Simpson E, Johns A, Putman M, Sasano H, Carr B. Aromatase expression in endometriosis: biology and clinical perspectives. Understanding and managing endometriosis: advances in research and practice. Quebec City: Parthenon Publishing; 1998.

45. Bulun SE, Zeitoun KM, Takayama K, Sasano H. Molecular basis for treating endometriosis with aromatase inhibitors. Hum Reprod Update. 2000;6:413-8.

46. Nawathe A, Patwardhan S, Yates D, et al. Systematic review of the effects of aromatase inhibitors on pain associated with endometriosis. BJOG. 2008;115:818-22.

47. Ferrero S, Venturini PL, Ragni N, et al. Pharmacological treatment of endometriosis: experience with aromatase inhibitors. Drugs. 2009;69:943-52.

48. Fang Z, Yang S, Gurates B, et al. Genetic or enzymatic disruption of aromatase inhibits the growth of ectopic uterine tissue. J Clin Endocrinol Metab. 2002;87:3460-6.

49. Noble LS, Takayama K, Zeitoun KM, et al. Prostaglandin E2 stimulates aromatase expression in endometriosis-derived stromal cells. J Clin Endocrinol Metab. 1997;82:600-6.

50. Vigano P, Mangioni S, Odorizzi MP, et al. Use of estrogen antagonists and aromatase inhibitors in endometriosis. Curr Opin Investig Drugs. 2003;4:1209-12.

51. Casper RF. Letrozole versus clomiphene citrate: which is better for ovulation induction? Fertil Steril. 2009;92:858-9.

52. Soysal S, Soysal ME, Ozer S, et al. The effects of post-surgical administration of goserelin plus anastrozole compared to goserelin alone in patients with severe endometriosis: a prospective randomized trial. Hum Reprod. 2004;19:160-7.

53. Ailawadi RK, Jobanputra S, Kataria M, et al. Treatment of endometriosis and chronic pelvic pain with letrozole and norethindrone acetate: a pilot study. Fertil Steril. 2004;81:290-6.

54. Amsterdam LL, Gentry W, Jobanputra S, et al. Anastrazole and oral contraceptives: a novel treatment for endometriosis. Fertil Steril. 2005;84:300-4.

55. Remorgida V, Abbamonte HL, Ragni N, et al. Letrozole and norethisterone acetate in rectovaginal endometriosis. Fertil Steril. 2007;88:724-6.

56. Ferrero S, Camerini G, Seracchioli R, et al. Letrozole combined with norethisterone acetate compared with norethisterone acetate alone in the treatment of pain symptoms caused by endometriosis. Hum Reprod. 2009;24:3033-41.

57. American College of Obstetricians and Gynecologists. ACOG Committee Opinion. Aromatase inhibitors in gynecologic practice. Obstet Gynecol. 2008;112:405-7.

58. Near AM, Wu AH, Templeman C, et al. Progesterone receptor gene polymorphisms and risk of endometriosis: results from an international collaborative effort. Fertil Steril. 2011;95:40-5.

59. Aghajanova L, Velarde MC, Giudice LC. The progesterone receptor coactivator Hic-5 is involved in the pathophysiology of endometriosis. Endocrinology. 2009;150:3863-70.

60. Kao LC, Germeyer A, Tulac S, et al. Expression profiling of endometrium from women with endometriosis reveals candidate genes for disease-based implantation failure and infertility. Endocrinology. 2003;144:2870-81. 
61. Bulun SE, Cheng YH, Yin $\mathrm{P}$, et al. progesterone resistance in endometriosis: link to failure to metabolize estradiol. Mol Cell Endocrinol. 2006;248:94-103.

62. Aghajanova L, Tatsumi K, Horcajadas JA, et al. Unique transcriptome, pathways and networks in the human endometrial fibroblast response to progesterone in endometriosis. Biol Reprod. 2011;84:801-15.

63. Bruner-Tran KL, Zhang Z, Eisenberg E, et al. Down-regulation of endometrial matrix metalloproteinase- 3 and -7 expression in vitro and therapeutic regression of experimental endometriosis in vivo by a novel nonsteroidal progesterone receptor agonist, tanaproget. J Clin Endocrinol Metab. 2006;91:1554-60.

64. Nayyar T, Bruner-Tran KL, Piestrzeniewicz-Ulanska D, et al. Developmental exposure of mice to TCDD elicits a similar uterine phenotype in adult animals as observed in women with endometriosis. Reprod Toxicol. 2007;23:326-36.

65. Vercellini P, Fedele L, Pietropaolo G, et al. Progestogens for endometriosis: forward to the past. Hum Reprod Update. 2003;9:38796. 